ISSN: $1576-0162$

DOI: http://dx.doi.org/10.33776/rem.v0i55.4679

\title{
PoBreZA Y EXCLUSIÓN SOCIAL EN LA UnIŌN EUROPEA
}

Poverty and Social Exclusion In the EuROPEAN Union

Pablo Podadera Rivera

Universidad de Málaga

ppodadera@uma.es

Ángel Romero Martín-Gambero

Universidad de Málaga

angelrmgambero@gmail.com

Antonio García Lizana

Universidad de Málaga

aglizana@uma.es

José Lobato Martín

Universidad de Málaga

jlobato@uma.es

Recibido: marzo de 2020; aceptado: abril de 2020

\section{RESUMEN}

Tras estudiar la situaciōn de la población en riesgo de pobreza o en exclusiōn social en los países de la Unión Europea, se indagan las variables que pueden tener influencia sobre dicha situaciōn, revisando las políticas adoptadas por las autoridades europeas, con el propósito de analizar su coherencia con las variables explicativas detectadas.

Palabras clave: AROPE; pobreza; exclusiōn social; Uniōn Europea; path analysis.

\section{ABSTRACT}

After studying the situation of population at risk of poverty or social exclusion in the countries of the European Union, the variables that can influence this situation are investigated, reviewing the policies adopted by the European authorities, with the aim of analyzing their coherence with the explanatory variables identified.

Keywords: AROPE; poverty; social exclusion; European Union; path analysis.

Clasificación JEL / JEL classification: C51; F02; 132. 



\section{INTRODUCCIŌN}

Desde hace décadas, pobreza y exclusión social son fenómenos de gran interés para las Ciencias Económicas. Señal de ello es la concesión del Premio Nobel de Economía a autores como Amartya Sen (1998) o Angus Deaton (2015).

Dicho interés parece haberse acentuado en años recientes, debido a los graves problemas económicos vividos. Pero incluso en épocas de bonanza económica, pobreza y exclusión han estado presentes. Conocer, por tanto, la situación es de gran importancia. Pero, asimismo, determinar los factores que influyen, condicionando el éxito de las intervenciones políticas.

Nuestro trabajo tiene tres objetivos: conocer la situación existente en los países de la UE; identificar aquellas variables que pueden influir sobre la misma; y revisar las políticas propuestas desde la UE, para determinar su idoneidad.

La metodología aplicada es la siguiente:

Para la medición de la pobreza y la exclusión, utilizamos la tasa de población en riesgo de pobreza o en exclusiōn social, elaborada por EUROSTAT, conocida como AROPE, según las siglas en inglés referidas a esa poblaciōn lat risk of poverty or exclusion). Para este indicador, se consideran personas en riesgo de pobreza o en exclusiōn social aquellas que están en algunas de estas situaciones:

Poblaciōn en riesgo de pobreza (TRP). Cuando viven en hogares cuya renta disponible total equivalente es inferior al umbral de riesgo de pobreza, fijado en el $60 \%$ de la mediana de la renta disponible equivalente nacional.

Poblaciōn sufriendo privaciōn material severa (PMS). Personas que no pueden hacer frente a cuatro de las siguientes situaciones: pagar el alquiler y las facturas corrientes; mantener la vivienda a una temperatura adecuada; permitirse una semana de vacaciones fuera de casa una vez al año; permitirse una comida de carne, pescado o proteína equivalente cada dos días; capacidad para afrontar gastos inesperados; disponibilidad de teléfono; televisión en color; lavadora; y automóvil.

Poblaciōn con Baja Intensidad de Trabajo en el Hogar (BITH). Personas de cero a 59 años, que reside en hogares en los que sus miembros en edad de trabajar (18 a 59 años) lo hicieron menos del 20\% de su potencial de trabajo durante el año anterior al de la entrevista. 
AROPE no es la suma de estos componentes, sino un indicador tridimensional, ya que pueden producirse intersecciones entre los componentes, por existir sujetos que participan simultáneamente de dos o tres de las situaciones indicadas. El componente BITH se calcula como porcentaje de la poblaciōn menor de 60 años, mientras que TRP y PMS son porcentajes del total de la poblaciōn. (Ver Esteban y Losa, 2015: 18-19).

Para identificar las variables explicativas que influyen sobre AROPE y su grado de incidencia, aplicamos la técnica econométrica denominada path analysis o análisis de influencias.

Finalmente, analizamos las políticas aplicadas, observando la coherencia de su contenido con las variables explicativas identificadas.

\section{Planteamiento del problema}

Si tenemos en cuenta el compromiso de la UE en la lucha contra la pobreza y la exclusión desde 1972; y, especialmente, desde 2000, no son de extrañar los numerosos estudios realizados sobre dichos problemas (Atkinson y Davoudi, 20001; Caballero y Cruz, 2011; González y otros, 2013; García y otros, 2016; Arroyo, 2016; Böhnke y Link, 2017...); sus relaciones con el crecimiento (ver Cruz y otros, 2017) y la recesión (Laparra, 2010; Fernández y otros, 2013); y las políticas aplicadas (por ej., Jones, 2010).

Dentro de este último grupo, podemos distinguir tres apartados, según el problema identificado (García y otros, 2016):

Compromiso político sesgado, excesivamente enfocado en el objetivo del crecimiento y en el establecimiento de indicadores, pero sin un firme apoyo financiero (Arriola, 2012 y 2014); asī como actuaciones verticales realizadas por los distintos Estados miembros, con objetivos diferentes, lo que plantea la necesidad de una reorientaciōn hacia prioridades y objetivos comunes (Martínez López, 2011; Ayala, 2012; Fresno y otros, 2012; Leschke y otros, 2012; Frazzer y otros, 2014).

Limitaciones metodológicas del indicador elegido (Laparra, 2010; Frazzer y otros, 2014; Ward y Ozdemir, 2013; Nolan y Whelan, 2011), referidas a diferencias temporales en los indicadores o en los ámbitos de referencia del umbral de los mismos, entre otros.

No atender la dimensión territorial del problema (Lois y otros, 2013; Athanasoglou y Dijkstra, 2014; Comité de las Regiones, 2014; Dijkstra y Athanasoglou, 2015).

Por nuestra parte, pretendemos profundizar en la lógica que subyace tras dicha dimensión, estudiando los factores que pueden explicar las diferencias existentes; y que, por tanto, deberían situarse en el punto de mira de las

${ }^{1}$ El artículo analiza el concepto de exclusión social y su utilidad para la política social europea, superando las limitaciones del concepto de pobreza, incluyendo otras dimensiones que enriquecerían la acciōn política; como, de hecho, ha ocurrido en los años posteriores a la publicaciōn. 
políticas, para incidir en las raíces, y no sólo en los síntomas2. Existen, a este respecto, diversos planteamientos. Asī, Kunze y Suppa (2017), utilizando como fuente el panel de datos alemán, examinan el impacto del desempleo sobre la participación social. Aun cuando este concepto no se corresponde exactamente con el de población en riesgo de pobreza o en exclusión social, puede tener ciertas concomitancias con un concepto ampliado de exclusión. Haataja (1999), a su vez, busca directamente la relación entre tasa de desempleo y pobreza; concluyendo que el riesgo de pobreza de los hogares en edad laboral no parece estar directamente relacionado con dicha tasa. Sin embargo, Garzón y Picatoste (2017) encuentra una estrecha conexión entre tasa de empleo y AROPE en España.

Otros autores, como Poza y Fernández (2011), explican la pobreza multidimensional en España como resultado de la precariedad de la situación laboral (el tipo de contrato es la variable con mayor poder explicativo), nivel de formaciōn, salud, condiciōn de inmigrante extracomunitario, falta de relaciones sociales, tipo de hogar (hogares monoparentales). Hernández (2010) señala los aspectos educativos como factor determinante en los procesos de pobreza y exclusión. Subirats y otros (2004), estudiando la realidad española y europea con variables "microeconómicas", tomando en cuenta edad, sexo, lugar de nacimiento, composición de hogar y otras de diferentes ámbitos (económico, laboral, formativo, sociosanitario, residencial, relacional), señalan que el primer factor en incidencia sobre la exclusión social es el desempleo desprotegido: personas que se encuentran desempleadas y no reciben ningún tipo de prestaciōn económica. Gomá y otros (2005), también efectúan un análisis de amplio espectro, considerando factores de carácter económico, laboral, formativo, sociosanitario, relacional, político, residencial y territorial.

En nuestro caso, estudiamos las condiciones que caracterizan cada territorio, como estructura productiva, demográfica, educativa, laboral y otras, ya que en la medida en que afectan al número de perceptores de renta, nivel de ingresos y condiciones de vida, pueden explicar las diferencias existentes (ver García y otros, 1989; Equipo de Investigaciōn Economía Cuantitativa del Bienestar, 1996).

\footnotetext{
2 No obstante, conviene tener presente la importancia concedida por algunos autores a las políticas que inciden directamente sobre los síntomas. Así, Haataja (1999) investiga el papel de las transferencias en la prevención de la pobreza en diferentes modelos de política social (Finlandia, Suecia, Dinamarca, Canadá, Alemania, Inglaterra, Estados Unidos y Australia), concluyendo que el modelo adoptado es esencial. Garzón y Picatoste (2017) analizan, a su vez, las políticas de austeridad ante la crisis económica en España, encontrando que la pérdida de poder adquisitivo de los salarios, al afectar a las rentas medias del hogar, influye en el aumento de AROPE.
} 
3. LAS POLITICAS APLICADAS Y LA SITUACIŌN DE LA POBREZA Y EXCLUSIŌN SOCIAL EN LA UE

\section{1. LAS POLITICAS APLICADAS: LIMITACIONES}

Desde la Cumbre de Paris de 1972 en que, por primera vez, se pone acento en la preocupación de las mejoras sociales como acompañante indiscutible de la expansión económica, hasta la puesta en marcha de la Estrategia 2020, la política de erradicación de la pobreza y la exclusión social en el proceso de construcción de la Unión Europea ha pasado por varios hitos, cuyos retos y compromisos, si bien se han correspondido con las necesidades imperantes, no se han traducido siempre en verdaderos logros reales. En efecto, la persistencia de la pobreza y la exclusión social en la UE, a lo largo de los años, ha venido motivando la preocupación por dar una respuesta política consistente, cuyos óptimos resultados se hacen esperar.

Habrá que esperar al año 2000 para poder hablar de una verdadera política europea contra la pobreza y la exclusión social con la puesta en marcha de la Estrategia de Lisboa, que estableció como objetivo "tener un efecto decisivo en la erradicación de la pobreza para 2010”, cuyas prioridades de actuaciōn concretas en el ámbito social se recogieron en la Agenda Europea de Política Social 2000-2005 (Comisión Europea, 2000) y donde los protagonistas fueron los "Programas Nacionales de Inclusión", que funcionaron bajo el "Método de Coordinaciōn Abierta" para la consecuciōn de objetivos comunes para la Uniōn. En este sentido la Recomendaciōn de la Comisiōn 2008/867/CE, de 3 de octubre de 2008, sobre la inclusión activa de las personas excluidas del mercado laboral recogía como principales pilares: rentas suficientes y adecuadas, mercados de trabajo inclusivos y acceso a unos servicios de calidad.

Entre tanto, el Panel de Hogares de la Unión Europea (PHOGUE) y la "Income, Social Inclusion and Living Conditions (EU-SILC)" fueron dos logros importantes como herramientas estadísticas para el estudio y análisis del tema.

Pero ante el poco éxito para alcanzar el objetivo marcado de erradicación efectiva de la pobreza en el año 2010 (proclamado por la UE como año de la lucha contra la pobreza y la exclusión social), ante la verificación de un aumento de casi 40 millones de pobres entre los años 80 y primeros dos mil, se puso en marcha en dicho año la Estrategia Europea 2020, con el objetivo de lograr que Europa creciera no solo de forma inteligente y sostenible, sino además, integradora y estableciendo como objetivo cuantitativo al respecto la reducción de 20 millones de personas en situación de pobreza para el año 2020 (Comisiōn Europea, 2010a, 2010b, 2011).

Como marco de actuación se estableciō la Plataforma Europea contra la Pobreza y la Exclusión Social (European Comission, 2012), donde se recogen las medidas dirigidas a conseguir tal objetivo: transversalidad de la acción social en el resto de las políticas, fondos de la UE en apoyo de la inclusión social, promociōn de la innovaciōn social, colaboraciōn entre actores y 
aprovechamiento del potencial de la economía social, mejora de la coordinación de las políticas entre los Estados miembros. Dichas medidas pretenden ampliar el espectro de actuación de la política social, centrado exclusivamente en el empleo y la protección social en la etapa anterior, activando en esta ocasión la inclusiōn social desde distintas perspectivas como reducción de las desigualdades sanitarias y servicios sociales más eficientes, generalizar el acceso a la educación infantil y reducir el fracaso escolar, nuevas políticas de apoyo a los jóvenes, mejorar la integración de inmigrantes, soluciones de eficiencia energética o acceso a los servicios financieros, entre otros.

No es de extrañar que las nuevas orientaciones políticas se vieran completadas con la elaboración por EUROSTAT de un nuevo indicador con el que se pretendía reflejar la complejidad y multiplicidad de los factores de la pobreza y la exclusión social y medir en consecuencia de forma simultánea ambos problemas en cada país. Nos estamos refiriendo a AROPE.

Ante las dificultades para alcanzar el objetivo de reducir en 20 millones las personas en riesgo de pobreza para el año 2020, y la evoluciōn observada en las tasas de riesgo de pobreza, coincidiendo con la recesión iniciada en 2007-2008, a comienzos de 2013, la Comisión presentaba el Paquete de Inversiōn Social para el Crecimiento y la Cohesión (European Comission, 2013), con la intención de imprimir una mayor prioridad a la inclusión social, a través sistemas de protecciōn social más eficientes, acompañadas de una Recomendación destinada a combatir la pobreza infantil. La supervisión y coordinaciōn de estas nuevas políticas de inversión quedaría garantizada por su inclusión en el Semestre Europeo.

El 17 de noviembre de 2017, el Parlamento Europeo, el Consejo y la Comisión Europea proclamaron de forma conjunta el pilar europeo de derechos sociales en la Cumbre social del empleo justo y el crecimiento celebrada en Gotemburgo. Dicho pilar se convierte en el nuevo marco de referencia para evaluar los resultados de los Estados miembros en el ámbito social y del empleo con el fin de reforzar la convergencia en la zona euro. Conviene resaltar aquí la propuesta de la Comisión en 2018 del nuevo Marco Financiero Plurianual 2021-2027, aun en negociaciones cuando se escriben estas líneas, donde se recoge la puesta en marcha de un Fondo Social Europeo Plus que integra la Iniciativa de Empleo Juvenil, el Fondo de Ayuda Europea para las Personas Más Desfavorecidas, el Programa de la Uniōn Europea para el Empleo y la Innovación Social y el Programa de Salud, para poner en práctica los principios y derechos del pilar europeo de derechos sociales.

Conviene advertir que las políticas aplicadas han estado orientadas fundamentalmente a atender de manera inmediata los problemas existentes, como mejorar los ingresos de la población, vivienda, sanidad, empleo...

Pero los resultados dejan que desear, a tenor de todo lo que se ha ido viendo, incluidos los cambios introducidos con el propósito de corregir intervenciones anteriores. Parece obvio que no basta, por tanto, con atender los sintomas existentes, sino intervenir sobre las eventuales raíces, como se ha señalado más arriba. 


\subsection{Situaciôn de LA POBREZA Y LA EXCLUSIÓN SOCIAL EN LA UE}

Para estudiar la situación existente, centraremos la atención en dos fechas especialmente significativas en relación con el comportamiento económico reciente: 2007, cuando se quebró la tendencia expansiva de los primeros años del siglo XXI; y 2016, año en el que las dificultades económicas se estaban superando (ver tabla 1).

TABLA 1. DifERENCIAS AROPE 2007-2016

\begin{tabular}{|c|c|c|c|}
\hline Países UE & AROPE 2007 & AROPE 2016 & Diferencias \\
\hline Alemania & $20,60 \%$ & $19,70 \%$ & $-0,90 \%$ \\
\hline Austria & $16,70 \%$ & $18,00 \%$ & $1,30 \%$ \\
\hline Bélgica & $21,60 \%$ & $20,70 \%$ & $-0,90 \%$ \\
\hline Bulgaria & $60,70 \%$ & $40,40 \%$ & $-20,30 \%$ \\
\hline Chipre & $25,20 \%$ & $27,70 \%$ & $2,50 \%$ \\
\hline Croacia & & $27,90 \%$ & \\
\hline Dinamarca & $16,80 \%$ & $16,70 \%$ & $-0,10 \%$ \\
\hline Eslovenia & $17,10 \%$ & $18,40 \%$ & $1,30 \%$ \\
\hline Eslovaquia & $21,40 \%$ & $18,10 \%$ & $-3,30 \%$ \\
\hline España & $23,30 \%$ & $27,90 \%$ & $4,60 \%$ \\
\hline Estonia & $22,00 \%$ & $24,40 \%$ & $2,40 \%$ \\
\hline Finlandia & $17,40 \%$ & $16,60 \%$ & $-0,80 \%$ \\
\hline Francia & $19,00 \%$ & $18,20 \%$ & $-0,80 \%$ \\
\hline Grecia & $28,30 \%$ & $35,60 \%$ & $7,30 \%$ \\
\hline Hungría & $29,40 \%$ & $26,30 \%$ & $-3,10 \%$ \\
\hline Irlanda & $23,10 \%$ & $24,20 \%$ & $1,10 \%$ \\
\hline Italia & $26,00 \%$ & $30,00 \%$ & $4,00 \%$ \\
\hline Letonia & $35,10 \%$ & $28,50 \%$ & $-6,60 \%$ \\
\hline Lituania & $28,70 \%$ & $30,10 \%$ & $1,40 \%$ \\
\hline Luxemburgo & $15,90 \%$ & $19,80 \%$ & $3,90 \%$ \\
\hline Malta & $19,70 \%$ & $20,10 \%$ & $0,40 \%$ \\
\hline Paises Bajos & $15,70 \%$ & $16,70 \%$ & $1,00 \%$ \\
\hline Polonia & $34,40 \%$ & $21,90 \%$ & $-12,50 \%$ \\
\hline Portugal & $25,00 \%$ & $25,10 \%$ & $0,10 \%$ \\
\hline Reino Unido & $22,60 \%$ & $22,20 \%$ & $-0,40 \%$ \\
\hline República Checa & $15,80 \%$ & $13,30 \%$ & $-2,50 \%$ \\
\hline Rumanía & $47,00 \%$ & $38,80 \%$ & $-8,20 \%$ \\
\hline Suecia & $13,90 \%$ & $18,30 \%$ & $4,40 \%$ \\
\hline$U E-28$ & $24,50 \%$ & $23,50 \%$ & $-1,00 \%$ \\
\hline
\end{tabular}

Fuente: elaboraciôn propia a partir de datos extraídos de EUROSTAT. 
Como puede verse, la distancia entre los valores extremos de AROPE se ha recortado. En 2007 era de 46,8 puntos porcentuales, y en 2016 de 27,1. Sin embargo, persisten las desigualdades, aun cuando se hayan producido determinados cambios. Mientras que los Estados del Norte y Centro mantienen las mejores posiciones, los países del Este conservan, en general, una posiciōn más grave, a pesar de que sus tasas hayan mejorado. Los países del Sur, junto con Irlanda, R. U. y Bélgica, ocupan una posiciōn intermedia; si bien ha empeorado la situación en los países meridionales.

En el Gráfico 1, se recoge el indicador AROPE para los países de la UE en el año 2016.

Gráfico 1. IndicAdor AROPE. AÑo 2016

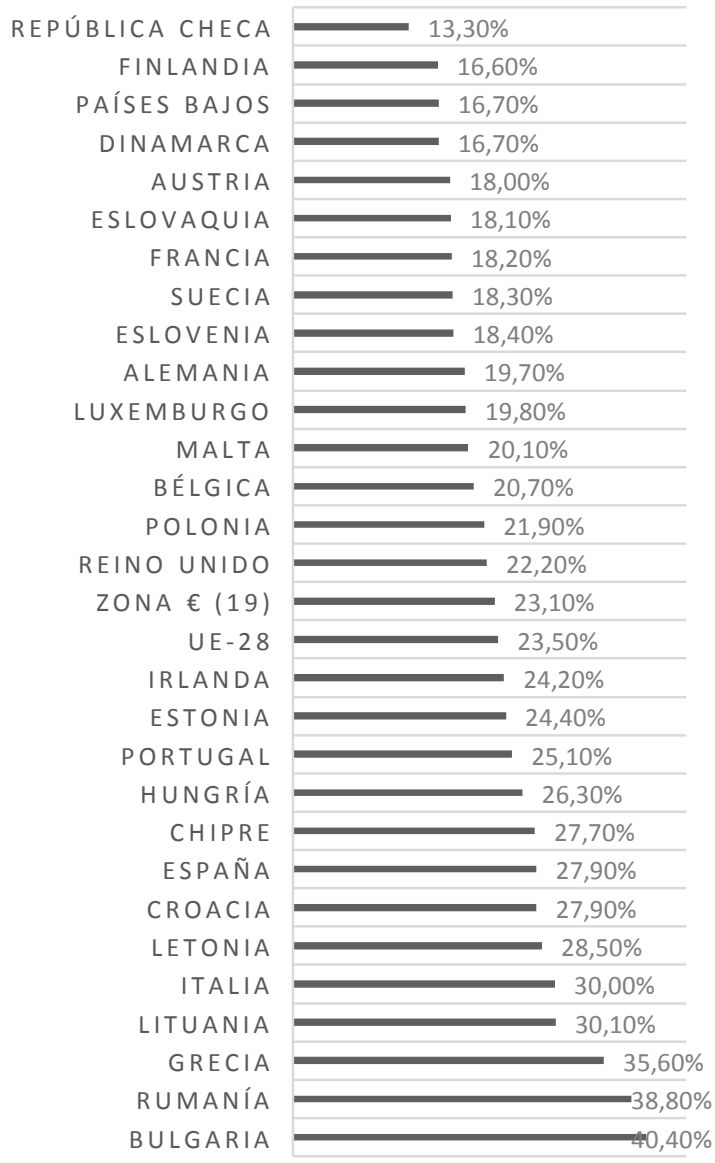

Fuente: EUROSTAT. Elaboraciōn propia. 
A la vista de los datos, podemos clasificar los países en tres grupos:

AROPE menor o igual al 20\%. República Checa, Finlandia, Paîses Bajos, Dinamarca, Austria, Eslovaquia, Francia, Suecia, Eslovenia, Alemania y Luxemburgo.

AROPE desde el 20\% al 30\%. Malta, Bélgica, Polonia, Reino Unido, Irlanda, Estonia, Portugal, Hungría, Chipre, España, Croacia, Letonia.

AROPE igual o superior al 30\%. Italia, Lituania, Grecia, Rumania, Bulgaria.

\section{El CONTEXTO SOCIOECONÓMICO dE LA POBREZA y LA EXCLUSIÓN SOCIAL. ANĀLISIS DE INFLUENCIAS}

Una vez observada la situaciōn, procede identificar y cuantificar qué variables explicativas pueden tener mayor incidencia sobre AROPE. Por razones analíticas; pero también políticas, para señalar las variables más apropiadas sobre las que intervenir para modificar la situaciōn. Con tales propósitos, se procederá a plantear un modelo que facilite dicha identificaciōn.

En este sentido, se utiliza un modelo econométrico; concretamente se opta por la especificación de un modelo multiecuacional de tipo recursivo, en el que sus ecuaciones planteadas son lineales, permitiendo la estimación de los parámetros de las diferentes ecuaciones por el método de mínimos cuadrados ordinarios.

Además se pretende aplicar el denominado análisis de influencias o path analysis. Esta técnica nos permitirá conocer otros datos importantes de la influencia de las variables explicativas sobre la explicada (ver Equipo de Investigaciōn Economía Cuantitativa del Bienestar, 1996).

El path analysis o análisis de influencias es un método multivariante que permite verificar el ajuste de modelos causales, asî como identificar la contribución directa e indirecta que realizan un conjunto de variables independientes para explicar la variabilidad de las variables dependientes. Además, es importante decir que esta técnica no prueba la causalidad, sino que sólo ayuda a seleccionar o inferir entre hipótesis causales (ver Pérez, Medrano y Sánchez, 2013: 52).

La aplicación del análisis de influencias mediante el modelo anteriormente mencionado, facilita la identificación de las variables que influyen sobre la pobreza y la forma en que tiene lugar su efecto sobre la misma: si de una forma directa o a través de otras variables.

Asimismo, conviene realizar algunas aclaraciones adicionales:

Este método permite diferenciar entre efectos directos, indirectos y totales. Los primeros serán aquellos que operan directamente de una variable sobre otra; los indirectos serān los que tienen lugar cuando la relaciōn entre dos variables es mediada por una o más variables. Los efectos totales de cada variable explicativa sobre la explicada se pueden descomponer, por tanto, en el efecto directo, más la suma de los efectos indirectos a través de las demás variables. La magnitud del efecto de la relación entre las variables se mide 
mediante los Ilamados coeficientes path. Hay que aclarar que el valor de los coeficientes path coincide con el valor de la estimación de los coeficientes del modelo recursivo lineal.

La representación gráfica del camino seguido hasta alcanzar la variable dependiente (en nuestro caso el indicador AROPE), a partir de las variables explicativas identificadas es lo que se conoce como diagrama path, en el que se recogen los efectos directos e indirectos de estas variables explicativas sobre la variable explicada, indicando sobre las líneas que vinculan a cada pareja de variables el correspondiente coeficiente path (ver Pérez, Medrano y Sánchez, 2013: 52-53).

De acuerdo con todo ello, el primer paso para la estimación del modelo, ha consistido en seleccionar posibles variables que permitan explicar la mayor o menor proporción de población en situación de riesgo de pobreza o en exclusión en cada país.

Teniendo en cuenta su posible incidencia, directa o indirecta, sobre la pobreza o la exclusiōn, de acuerdo con lo señalado en el epígrafe 2, se han seleccionado las variables siguientes:

Tasa de Actividad.

Tasa de Ocupación

Tasa de Paro.

Porcentaje de población que solo posee estudios primarios.

Tasa de Población con más de 65 años.

Tasa de Población que tiene entre 16 y 65 años.

Tasa Empleo Industrial.

Tasa Empleo Agrícola.

Tasa Empleo en Servicios.

Sin embargo, al construir el modelo econométrico planteado, se terminan excluyendo tres de las nueve variables consideradas inicialmente (la tasa de paro, la tasa de empleo industrial y la tasa de ocupación), puesto que al formular el modelo se consigue un mejor ajuste de las ecuaciones sin contar con ellas Por lo que se mantienen las variables siguientes:

TA: Tasa de Actividad.

TEA: Tasa de Empleo Agrario.

T_POB_SIN_ESTUD: Poblaciōn que solo posee estudios primarios.

TES: Tasa Empleo en Servicios.

P3: Poblaciōn mayor de 65 años.

P1: Población de entre 16 y 65 años.

Las correlaciones encontradas entre las variables socioeconómicas mencionadas permiten construir la tabla 2:

Los coeficientes de correlaciōn más altos y relevantes han sido los encontrados con la tasa de empleo agrario y el indicador de población en riesgo de pobreza o en exclusión social (0.642). El signo positivo está indicando que el porcentaje de empleo agrario se mueve de forma directa con el indicador AROPE; es decir, a mayores valores del porcentaje de empleo agrario, Ios países de la UE tienen una peor situaciōn en cuanto a pobreza o exclusiōn. Lo 
TABLA 2. Matriz de CoRrelaciones de LAS VARIABLES (2016)

\begin{tabular}{|l|r|r|r|r|r|r|r|}
\hline & AROPE & P1 & P3 & \multicolumn{1}{c|}{ TA } & \multicolumn{1}{c|}{$\begin{array}{c}\text { POB SIN } \\
\text { ESTUD }\end{array}$} & \multicolumn{1}{c|}{ TEA } & \multicolumn{1}{c|}{ TES } \\
\hline AROPE & 1.000000 & 0.055637 & 0.110429 & -0.245776 & 0.181122 & 0.642000 & -0.382008 \\
\hline P1 & 0.055637 & 1.000000 & -0.675246 & 0.244293 & -0.192902 & 0.176468 & -0.257333 \\
\hline P3 & 0.110429 & -0.675246 & 1.000000 & -0.477991 & 0.359823 & -0.029374 & -0.104626 \\
\hline TA & -0.245776 & 0.244293 & -0.477991 & 1.000000 & -0.505801 & -0.277316 & 0.378308 \\
\hline $\begin{array}{l}\text { POB SIN } \\
\text { ESTUD }\end{array}$ & 0.181122 & -0.192902 & 0.359823 & -0.505801 & 1.000000 & 0.021310 & 0.111702 \\
\hline TEA & 0.642000 & 0.176468 & -0.029374 & -0.277316 & 0.021310 & 1.000000 & -0.725002 \\
\hline TES & -0.382008 & -0.257333 & -0.104626 & 0.378308 & 0.111702 & -0.725002 & 1.000000 \\
\hline
\end{tabular}

Fuente: EUROSTAT, Banco Mundial, OIT. Elaboración propia.

que también ocurre con la tasa de población sin estudios, cuya relación con el indicador AROPE es también directa, como cabría esperar.

Por otro lado, hay que destacar la correlación negativa entre la tasa de empleo en servicios y el indicador AROPE (-0.382), lo cual resulta razonable porque a medida que un país se desarrolla, los servicios empiezan a tener un peso mayor en el conjunto de la producción; este proceso de terciarización va acompañado de una disminución de la participación del sector primario en la economía, implicando, además, un incremento de las rentas percibidas; aun cuando puedan existir matizaciones, según ramas de actividad. También ocurre con la tasa de actividad y el indicador AROPE $(-0,245)$; por tanto, a mayor tasa de empleo en servicios o mayor tasa de actividad, el indicador AROPE es más reducido.

Otra variable importante en su relaciōn con AROPE, aunque con un valor de su coeficiente de correlaciōn un poco inferior, es la poblaciōn que sólo tiene estudios primarios $(0,181)$. El signo positivo establece una nueva relación directa, lo cual parece razonable, ya que más poblaciōn sin estudios o solo con estudios primarios, implicaría menor posibilidad de percepciōn de ingresos suficientes que permita al individuo vivir con cierta sostenibilidad y estabilidad.

La utilizaciōn de un modelo econométrico da una mayor consistencia al estudio. Se opta por la especificaciōn de tres ecuaciones, donde las variables dependientes o endógenas serān AROPE (tasa de poblaciōn en riesgo de pobreza o en exclusión social), la tasa de actividad (TA) y la tasa de empleo agrario (TEA).

En la primera ecuación se explica el indicador AROPE en función de la tasa de actividad, la tasa de empleo agrario y la población que sólo tiene estudios primarios, mientras que las otras dos ecuaciones estimadas tratan de explicar determinados aspectos del mercado de trabajo.

En la segunda ecuación, la tasa de actividad definida explica de una manera más concreta cómo es influida por la tasa de empleo agrario (TEA), la poblaciōn sin estudios o estudios primarios (T_POB_SIN_ESTUD), la tasa de empleo en el sector servicios (TES) y la poblaciōn mayor de 65 años (P3). 
Y por último, en la tercera ecuación se explica la tasa de empleo agrario en función de la población sin estudios o estudios primarios, la tasa de empleo en el sector servicios, la población mayor de 65 años y la población de entre 16 y 65 años (P1).

El modelo, en cuestión, queda reflejado por las relaciones anteriormente descritas:

(1) $A R O P E=f\left(T A, T E A, T \_P O B \_S I N \_E S T U D\right)$

(2) $T A=f(T E A, T$ POB_SIN_ESTUD), TES, P3)

(3) $\left.T E A=f\left(T \_P O B \_S I N \_E S T U D\right), T E S, P 3, P 1\right)$

La aplicaciōn de las técnicas del análisis de influencias permite el uso de un modelo multiecuacional recursivo; en este tipo de modelos, las variables endógenas dependen además de sus variables predeterminadas, de otras endógenas, pero sin existir relaciones recíprocas de causalidad, y además, no hay correlaciōn contemporánea entre los términos de perturbación de las distintas ecuaciones; es decir, $\operatorname{cov}(u 1 t, u 2 t)=\operatorname{cov}(u 1 t, u 3 t)=\operatorname{cov}(u 2 t$, $\mathrm{u} 3 \mathrm{t})=0$.

En el diseño del sistema de ecuaciones que se plantea en este estudio, la primera circunstancia se cumple; sólo faltaría comprobar para que efectivamente se trata de un modelo recursivo y no de un modelo de ecuaciones simultáneas, la ausencia de correlación entre las perturbaciones de las diferentes ecuaciones.

La estimaciōn de las ecuaciones anteriores se podrá llevar a cabo por el método de mínimos cuadrados ordinarios (MCO) si se cumple el supuesto de no correlaciōn entre las variables explicativas y la parte estocástica; para ello, las covarianzas entre las distintas perturbaciones aleatorias deben ser nulas, circunstancia que comprobaremos mediante un contraste de hipótesis. Teniendo en cuenta, que la estimaciōn por MCO de cada una de las ecuaciones por separado, proporcionan los mismos resultados que si se procediera a la estimación del sistema de forma conjunta.

La estimaciōn por MCO de cada una de las ecuaciones del modelo se recoge en la tabla 3.

Las cifras que figuran entre paréntesis reflejan la probabilidad del estadístico t de Student, correspondiente a un contraste individual de los coeficientes

Tabla 3. Estimaciōn del modelo Recursivo por MCO

\begin{tabular}{|l|r|r|r|r|r|r|r|c|}
\hline & TA & TEA & $\begin{array}{r}\text { T_POB_SIN_ } \\
\text { ESTUD }\end{array}$ & P3 & P1 & TES & R2 & P (F) \\
\hline AROPE & $\begin{array}{r}0.0219 \\
(0.8952)\end{array}$ & $\begin{array}{r}0.8555 \\
(0.0003)\end{array}$ & $\begin{array}{r}0.0989 \\
(0.3060)\end{array}$ & & & & 0.4406 & 0,0015 \\
\hline TA & & $\begin{array}{r}0.0474 \\
(0.8775)\end{array}$ & $\begin{array}{r}-0.2744 \\
(0.0070)\end{array}$ & $\begin{array}{r}-0.8539 \\
(0.0980)\end{array}$ & & $\begin{array}{r}0.3557 \\
(0.0587)\end{array}$ & 0.5113 & 0,0009 \\
\hline TEA & & & $\begin{array}{r}0.0813 \\
(0.1806)\end{array}$ & $\begin{array}{r}-0.7733 \\
(0.0996)\end{array}$ & $\begin{array}{r}-0.6081 \\
(0.2377)\end{array}$ & $\begin{array}{r}-0.4903 \\
(0.0000)\end{array}$ & 0.5848 & \\
\hline
\end{tabular}

Fuente: elaboración propia a partir del programa Eviews. 
del modelo, valores por encima de 0.05 nos lleva a no rechazar la hipótesis nula de no significatividad individual del parămetro, con una confianza del 95 $\%$, y por consiguiente la variable en cuestión resultaría no relevante para el modelo, mientras que la columna $\mathrm{P}(\mathrm{F})$ indica la probabilidad del estadístico $\mathrm{F}$ Fisher-Snedecor correspondiente al contraste de significatividad conjunta del modelo, de las tres ecuaciones que conforman el modelo.

El valor de la bondad del ajuste para cada una de las ecuaciones estimadas asciende a $0.44,0,51$ y 0.58 , respectivamente. De esta forma el $44 \%, 51 \%$ y $58 \%$ de las variaciones de las variables AROPE, TA y TEA, respectivamente, vienen explicados por las variables explicativas de dichas ecuaciones. Aunque las ecuaciones no tienen un excelente ajuste, se puede considerar aceptable y no muestran fuertes discrepancias entre los valores reales y ajustados por el modelo.

La no significatividad individual de la mayoría de los coeficientes y la significatividad conjunta del modelo, pueden ser indicios de problemas de multicolinealidad; para comprobarlo, se obtienen los factores inflacionarios de la varianza (FIV).

El FIV muestra la forma como la varianza de un estimador se infla por la presencia de multicolinealidad. A medida que el coeficiente de correlación entre dos regresores elevado al cuadrado, se acerca a 1, el FIV tiende a infinito. Es decir, a medida que el grado de colinealidad aumenta, la varianza de un estimador también y, en el límite, se vuelve infinita (Gujarati y Porter 2009, p. 328). Factores que aparecen en la tabla 4.

TABLA 4. FACTORES INFLACIONARIOS DE LA VARIANZA (FIV)

\begin{tabular}{|c|c|c|c|}
\hline (FIV) & Ecuación l (AROPE) & Ecuación 2 (TA) & Ecuación 3 (TEA) \\
\hline TA & 1.4856 & & \\
\hline TEA & 1.1060 & 2.2755 & \\
\hline T_PON_SIN_ESTUD & 1.3720 & 1.2429 & 1.2118 \\
\hline TES & & 2.357 & 1.326 \\
\hline P3 & & 1.2426 & 2.5198 \\
\hline P1 & & & 2.36608 \\
\hline
\end{tabular}

Fuente: elaboraciōn propia a partir del programa Eview.

Un valor superior a 10 de este factor, podría considerarse sintoma de un problema grave de multicolinealidad, cosa que no ocurre con el sistema de ecuaciones estimadas por este método. Esta situaciōn también podía reflejarse en la tabla 2, al no figurar correlaciones elevadas entre los distintos regresores del modelo.

Para comprobar la no existencia de correlación contemporánea entre las perturbaciones aleatorias de las diferentes ecuaciones, se opta por representar la matriz de correlaciones de los residuos mínimo cuadráticos de cada una de las ecuaciones propuestas. 
TABLA 5. MATRIZ DE CORRELACIONES DE LOS RESIDUOS MINIMOCUADRÁTICOS

\begin{tabular}{|c|c|c|c|}
\hline & AROPE & TA & TEA \\
\hline AROPE & 1.000000 & -0.006852 & 0.139645 \\
\hline TA & -0.006852 & 1.000000 & 0.041880 \\
\hline TEA & 0.139645 & 0.041880 & 1.000000 \\
\hline
\end{tabular}

Fuente: elaboraciōn propia a partir del programa Eviews.

Como vemos, esta matriz indica valores próximos a 0 en las correlaciones de los residuos; esto da una primera aproximación de indicios de no existencia de correlación contemporánea entre las perturbaciones, dando mayor solidez a que la formulación del modelo lineal recursivo inicialmente planteado es correcta.

No obstante, para mayor rigor en nuestro análisis, se decide aplicar un contraste que permita establecer conclusiones firmes en cuanto a la no existencia de correlaciones entre las distintas perturbaciones. Los resultados obtenidos confirmarían que las variables exógenas elegidas son estrictamente exógenas y no variables endógenas. De esta forma se confirmaría que el modelo utilizado es un modelo recursivo y no un modelo de ecuaciones simultáneas, y se evitaría utilizar otros métodos de estimación distintos a MCO. El método MCO proporcionaría estimaciones con buenas propiedades asintóticas, son consistentes.

En primer lugar, se procede a estudiar la exogeneidad de las variables TA (tasa de actividad) y TEA (tasa de empleo agrario) en la ecuaciōn de AROPE (Ecuación 1). Los resultados aparecen en la Tabla 6.

Tabla 6. Contraste de endogenidad de TA y TEA en ECuAciōn 1

\begin{tabular}{|l|}
\hline Test de Endogeneidad \\
\hline Hipótesis nula: TA TEA son exōgenas \\
\hline Especificaciôn: AROPE C TA TEA T_POB_SIN_ESTUD \\
\hline Especificaciôn de instrumentos: C TEA T_POB_SIN_ESTUD P3 P1 \\
\hline Variables endōgenas para tratar como exôgenas: TA TEA \\
\hline Valor Grados Lib. Probabilidad \\
\hline Diferencia del estadístico J 0.38792410 .5334 \\
\hline
\end{tabular}

Fuente: elaboraciōn propia a partir del programa Eviews.

La probabilidad del estadístico correspondiente al incremento de J nos lleva a no rechazar la hipótesis nula con una confianza del 95\%, por tanto, TA y TEA son variables exōgenas en la ecuaciōn de AROPE.

En segundo lugar, se estudia la exogeneidad de la variable TEA (tasa de empleo agrario) en la ecuación de la TA (tasa de actividad) (Ecuación 2). Los resultados aparecen en la Tabla 7. 
Tabla 7. Contraste de endogenidad de TEA en ECUaciōn 2

\begin{tabular}{|l|}
\hline Test de Endogeneidad \\
\hline Hipôtesis nula: TEA es exōgena \\
\hline Especificaciôn: TA C TEA T_POB_SIN_ESTUD TES P3 \\
\hline Especificaciôn de instrumentos: C TEA T_POB_SIN_ESTUD P3 P1 \\
\hline Variables endógenas para tratar como exōgenas: TEA \\
\hline Valor Grados Lib Probabilidad \\
\hline Diferencia del Estadístico J 0.79290010 .3732 \\
\hline
\end{tabular}

Fuente: elaboraciōn propia a partir del programa Eviews.

Nuevamente, el estadístico J confirma que la variable TEA (tasa de empleo agrario) es una variable exógena en la ecuaciōn 2, con una confianza del 95\%. Se confirma por tanto, la no correlaciōn entre las variables explicativas de cada ecuación y la perturbación aleatoria correspondiente. Consecuentemente la matriz de varianzas y covarianzas de las perturbaciones estructurales, es un matriz diagonal, debido a las covarianzas nulas entre las perturbaciones de cada una de las tres ecuaciones. La aplicaciōn del análisis de influencias mediante este modelo recursivo y no por un modelo de ecuaciones simultáneas, para estudiar la situación de pobreza y exclusiōn social en los países de la Unión Europea ha resultado ser correcta, según los resultados obtenidos.

Una vez realizadas las anteriores comprobaciones podemos pasar a estudiar los efectos que tienen las variables explicativas sobre la explicada; teniendo en cuenta, como más arriba se ha señalado, la diferencia establecida entre efectos totales y parciales, tal como se muestra en la tabla 8.

Una vez que hemos calculado los efectos directos e indirectos, hemos podido obtener los efectos totales; de esta manera se pueden determinar las variables que tienen un mayor impacto sobre indicador AROPE (con independencia de que el efecto sea positivo o negativo), las cuales expuestas de forma ordenada por orden descendente, son las siguientes:

TEA $(0.8555806)$

P3 $(-0.680216)$

P1 $(-0.520224)$

TES $(-0.411645)$

T_POB_SIN_ESTUD (0.162495)

TA (0.021873).

Para indicar la red de influencias detectada, en la figura 2 podemos observar el correspondiente diagrama path (ver Fig. 2).Como puede observarse, destaca en especial por su incidencia sobre AROPE la estructura productiva existente, identificada en el modelo a través de la tasa de empleo en los sectores primario y servicios. Sobre todo, es de subrayar el peso que representa en términos de influencia la tasa de empleo agrícola, lo que es coherente con las características de dicho empleo, las rentas percibidas, 
Tabla 8. Efectos totales y parciales

\begin{tabular}{|c|c|c|}
\hline Variable & Efecto & Cuantia \\
\hline$T \_P O B \_S I N \_E S T U D$ & $\begin{array}{r}\text { Directo } \\
\text { Vía TA } \\
\text { Vía TEA } \\
\text { Total }\end{array}$ & $\begin{array}{r}0.098990 \\
-0.006003 \\
0.069508 \\
0.162495\end{array}$ \\
\hline P3 & $\begin{array}{r}\text { Directo } \\
\text { Vía TA } \\
\text { Vĩa TEA } \\
\text { Total }\end{array}$ & $\begin{array}{r}0 \\
-0.018678 \\
-0.661538 \\
-0.680216\end{array}$ \\
\hline TES & $\begin{array}{r}\text { Directo } \\
\text { Vía TA } \\
\text { Vía TEA } \\
\text { Total }\end{array}$ & $\begin{array}{r}0 \\
0.007781 \\
-0.419426 \\
-0.411645\end{array}$ \\
\hline Pl & $\begin{array}{r}\text { Directo } \\
\text { Vía TA } \\
\text { Vía TEA } \\
\text { Total }\end{array}$ & $\begin{array}{r}0 \\
0 \\
-0.520224 \\
-0.520224\end{array}$ \\
\hline$T A$ & Directo (total) & 0.021873 \\
\hline TEA & $\begin{array}{r}\text { Directo } \\
\text { Vía TA } \\
\text { Total }\end{array}$ & $\begin{array}{r}0.855477 \\
0.0001036 \\
0.8555806\end{array}$ \\
\hline
\end{tabular}

Fuente: elaboración propia.

las condiciones del hábitat rural, etc. De ahí la necesidad de modificar la estructura productiva en los países condicionados por las tasas más levadas de AROPE, de manera que se reduzca el peso del sector agrario en términos relativos. Políticas centradas en la mera protección social, sin ahondar en las raíces que subyacen en el fondo, podrían explicar los repetidos fracasos de los objetivos que sucesivamente se han venido marcando por las instituciones comunitarias, y el fracaso en el cumplimiento efectivo de los derechos sociales reconocidos.

FIGURA 2: DIAGRAMA PATH

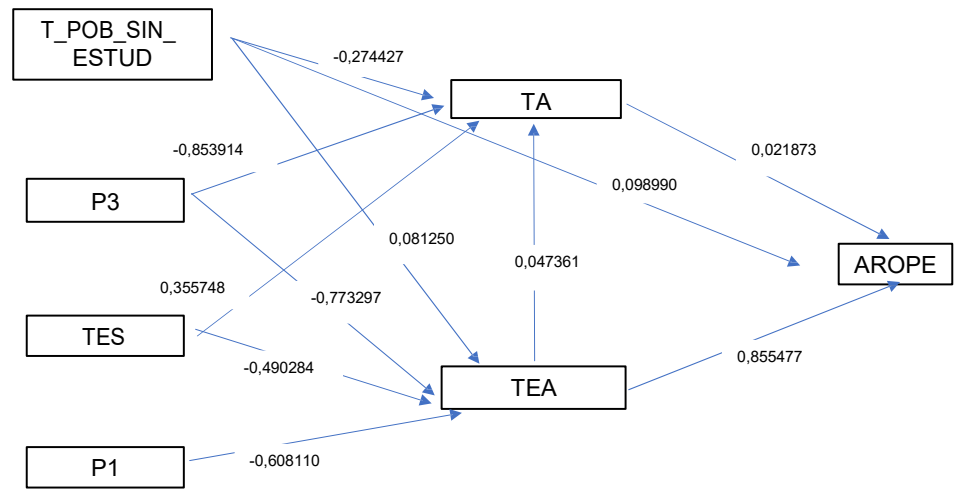

Fuente: elaboración propia. 


\section{CONCLUSIONES}

La situación de los estados miembros de la UE en términos de riesgo de pobreza o exclusión social muestra una gran disparidad; manteniéndose en el tiempo una ordenaciōn bastante similar.

Tal distribuciōn territorial aparece vinculada especialmente con la estructura sectorial del empleo, teniendo en cuenta que la variable que tiene una mayor influencia positiva sobre el indicador AROPE es la tasa de empleo agrario; al mismo tiempo que la tasa de empleo en el sector servicios muestra un efecto negativo sobre AROPE. De donde se deduce la necesidad de llevar a cabo actuaciones que permitan modificar la estructura productiva, rebajando el peso del sector agrario y diversificando la actividad económica.

Frente a tal estado de cosas, desde 1972, las políticas contra la pobreza y la exclusión social han estado presentes en el proceso de construcción de la UE, definidas en torno a objetivos que se han ido renovando; nunca suficientemente alcanzados. Dichas políticas han estado caracterizadas, fundamentalmente, por atender los sintomas, y no las variables que presentan mayor influencia sobre la situaciōn; por lo que deben ser revisadas.

Obviamente, no se pretende dar por zanjado un tema que requiere estudios más amplios y diversificados; pero sí aportar algunas líneas de reflexiōn que ayuden a mejorar la situación.

\section{REFERENCIAS}

Abel Smith, B. y otros (1981): Final report from the Commision to the Council on the first programme of pilot schemes and studies to combat poverty, Commision of the European Communities, COM (81) 769. En http://aei. pitt.edu/12274/.

Arriola, J. (2012): "Europa 2020: una estrategia contra la pobreza en vía muerta"; en Comité Técnico de la Fundaciōn FOESSA (coord.): Exclusión y Desarrollo Social, Fundación FOESSA/Cáritas, Madrid.

Arriola, J. (2014): "La estrategia de la Unión Europa de lucha contra la pobreza". VII Informe sobre Exclusión y Desarrollo Social en España. Documento de trabajo 8.2, Fundaciōn FOESSA.

Arroyo, M. (2016): "Exclusiōn y pobreza en la Uniōn Europea”, GeoGraphos: Revista Digital para Estudiantes de Geografia y Ciencias Sociales, 7 (85), 104-131.

Athanasoglou, S. y Dijkstra, L. (2014): The Europe 2020 Regional Index. Science and Policy Report. Publications Office of the European Union.

Atkinson, R. y Davoudi, S. (2000): "The Concept of Social Exclusion in the European Union: Context, Development and Possibilities," Journal of Common Market Studies, 38(3), 427-448. 
Ayala, L. (2010): “ ¿Es la política social la "cenicienta” de la crisis? Retos y políticas para la inclusiōn social”; en EAPN: Nuevas propuestas para nuevos tiempos, EAPN, Madrid.

Böhnke, P. y Link, S. (2017): "Poverty and the Dynamic of Social Networks. An Analysis of German Panel Data”, European Sociological Review, 33 (4), 615-632.

Caballero Fernández, R. y Cruz Morato, M. A. (2011): “Análisis de la exclusiōn social en la Uniōn Europea basado en indicadores sintéticos", Revista Electrónica de Comunicaciones y Trabajos de ASEPUMA, 12, 85-104.

CES (2016): La política europea contra la pobreza y la exclusión social: avances y retos. Cauces: Cuadernos del Consejo Económico y Social, ISSN 1888038X, ISSN-e 1988-3463, Nº. 32, págs. 31-41.

Comisión de las Comunidades Europeas (2003): Informe conjunto sobre la inclusión social en el que se resumen los resultados del examen de los planes nacionales de acción en favor de la inclusión social (2003-2005), Comisiōn de las Comunidades Europeas, COM (2003) 773. Disponible en: https:// eur-lex.europa.eu/legal-content/ES/TXT/?uri = CELEX:52003DC0773.

Comisiōn Europea (2000): Agenda de política social, COM (2000) 379.

Comisiōn Europea (2009): Una estrategia de la UE para la juventud: inversión y capacitación Un método abierto de coordinación renovado para abordar los desafios y las oportunidades de los jóvenes, COM (2009) 200 final.

Comisión Europea (2010a): EUROPA 2020 Una estrategia para un crecimiento inteligente, sostenible e integrador, COM (2010) 2020 final.

Comisión Europea (2010b): La Plataforma Europea contra la Pobreza y la Exclusión social: Un marco europeo para la cohesión social y territorial, COM (2010) 758 final.

Comisión Europea (2011): La dimensión social de la estrategia Europa 2020. Un informe del comité de protección social. Oficina de Publicaciones de la Unión Europea, Luxemburgo.

Comisiōn Europea (2017): Priority Policy Area. Pilar Europeo de Derechos Sociales. Construir una Unión Europea más inclusiva y justa. En https:// ec.europa.eu/commission/priorities/deeper-and-fairer-economic-andmonetary-union/european-pillar-social-rights_es

Comité de las Regiones (2014): Blueprint for a Revised Europe 2020 Strategy.

Cruz Morato, M. A., García Lizana, A. y García Mestanza, J. (2017): “El impacto de la exclusión social sobre el crecimiento económico en la Uniōn Europea”, Revista de Economía Mundial, 45, 43-64.

Deaton, A. (2013): El Gran Escape. Salud, riqueza y los orígenes de la desigualdad. Fondo de Cultura Económica de España, Madrid.

Departamento de Asuntos Económicos y Sociales (1999): Códigos uniformes de país o de zona para uso estadístico, Organización de las Naciones Unidas. En https://unstats.un.org/unsd/methodology/m49/.

Dijkstra, L. y Athanasogolou, S. (2015): The Europe 2020 Index: The Progress of EU Countries Regions and Cities to the 2020 Targets. Regional Focus, $1 / 2015$. 
Equipo de Investigaciōn Economía Cuantitativa del Bienestar (1996): La medición de la pobreza en Andalucía, Facultad de Ciencias Económicas y Empresariales, Málaga.

Esteban Yago, M.A. y Losa Carmona, A. (2015): Guía básica para interpretar los indicadores de desigualdad, pobreza y exclusión social, EAPN España, Madrid. En https://www.eapn.es/publicaciones/235/guia-basica-parainterpretar-los-indicadores-de-desigualdad-pobreza-y-exclusion-social.

European Comission (2012): Investing in a Social Europe. Report on the Second Annual Conference of the European Platform against Poverty and Social Exclusion. Bruselas, 5-7 diciembre 2012

European Comission (2013): Towards Social Investment for Growth and Cohesion- Including Implementing the European Social Fund 2014-2020, COM (2013) 83 final.

Fernández Morales, A., García Lizana, A. y Martín Reyes, G. (2013): "Pobreza y recesión en la zona euro", Revista de Economía Mundial, 32, 153-178.

Frazer, H., Guio, A. C., Marlier, E., Vanhercke, B. y Ward, T. (2014): Putting the Fight against Poverty and Social Exclusion at the Heart of the EU Agenda: A Contribution to the Mid-Term Review of the Europe 2020 Strategy. OSE Research Paper, 15.

Fresno, J.M., Renes Ayala, V. y Tsolakis, A. (2012): “Estrategia Europa 2020 e inclusión social: distanciamiento creciente entre objetivos, políticas e instrumentos", Zerbitzuan: Revista de Servicios Sociales, 51, 27-47.

García Lizana, A., Martín Reyes, G., Ladoux Aguilar, M. y Fernández Morales, A. (1989): "Employment, Unemployment and Poverty in Spain”, XV European Symposium on Social Welfare, Noordwijkerhout.

García Luque, O., Faura Martínez, U. y Lafuente Lechuga, M. (2016): "Objetivo Europa 2020. La reducciōn de la pobreza y la exclusión social en España”, Papers: Revista de Sociología, 101 (4), 503-526.

Garzōn Gordōn, A. J. y Picatoste, J. (2017): "Políticas de austeridad para afrontar la crisis económica en España: la pérdida de poder adquisitivo de las rentas salariales y el aumento de las desigualdades", XXIV Encuentro de Economía Pública, Toledo.

Gomá Carmona, R., Subirats i Humet, J., Brugué Torruella, J. (2005): Análisis de los factores de exclusión social. Documentos de Trabajo. Fundación BBVA.

Gujarati, D.N. y Porter D.C. (2009): Basics Econometrics, McGraw-Hill, Nueva York, (1 ${ }^{\text {a }}$ ed. 1978).

Haataja, A. (1999): "Unemployment, Employment and Poverty", European Societies, 1 (2), 169-196.

Hernández Pedreño, M. (2010): "El estudio de la pobreza y la exclusión social. Aproximaciōn cuantitativa y cualitativa”, Revista Interuniversitaria de Formación del Profesorado, 24 (3), 25-46.

Jones, S. (2010): “La estrategia Europa 2020. ¿Influirá en la pobreza?”, Revista de la Red Europea de Lucha contra la Pobreza y la Exclusión, 133, 6-7. 
Kunze, L. y Suppa, N. (2017): "Bowling Alone or Bowling at all? The Effect of Unemployment on Social Participation”, Journal of Economic Behavior \& Organization, 133, 213-235.

Laparra, M. (2010): "El impacto de la crisis en la cohesión social o el surf de los hogares españoles en el modelo de integraciōn de la sociedad líquida", Documentación Social, 158, 97-130.

Leschke, J., Theodoropoulou, S. y Watt, A. (2012): "How do Economic Governance Reforms and Austerity Measures Affect Inclusive Growth as Formulated in the Europe 2020 Strategy?". En Lehndorff, S. (ed.): A Triumph of Failed Ideas: European Models of Capitalism in the Crisis, European Trade Union Institute (ETUI), Bruselas.

Lois González, R. C., Feal Pérez, A. M. y Paül Carril, V. (2013): “La dimensiōn territorial de la Estrategia Europa 2020. Las regiones europeas en la senda oficial para salir de la crisis", Ería: Revista cuatrimestral de geografia, 92 , 211-242.

Martinez López, A. (2011): “El discurso de la Uniōn Europea en materia de políticas de empleo y exclusión social. Análisis sociológico de la Estrategia Europea de Empleo", Papers: Revista de Sociología, 96 (1), 35-54.

Nolan, B. y Whelan, C. T. (2011): The EU 2020 Poverty Target. GINI Discussion Paper, 19.

Pérez, E., Medrano, L.A. y Sánchez Rosas, J. (2013): "El Path Analysis: conceptos básicos y ejemplos de aplicación", Revista Argentina de Ciencias del Comportamiento, 5 (1), 52-66.

Poza Lara, C. y Fernández Cornejo, J. A. (2011): “¿Qué factores explican la pobreza multidimensional en España? Una aproximaciōn a travês de los modelos de ecuaciones estructurales", Revista de Métodos Cuantitativos para la Economía y la Empresa, 12, 81-110.

Sánchez Fernández, J. (2004): Introducción a la estadística empresarial. En http://www.eumed.net/cursecon/libreria/index.htm.

Sen, A. (1995): Nuevo examen de la desigualdad, Alianza Editorial, Madrid.

Subirats, J. (director) y otros (2004): Pobreza y exclusión social. Un análisis de la realidad española y europea. Fundación La Caixa, Barcelona.

Ward, T. y Ozdemir, E. (2013): "Measuring Low Work Intensity: An Analysis of the Indicator". ImPRovE Working Paper, 13/09. 
